\title{
Borboletas (Lepidoptera: Hesperioidea e Papilionoidea) visitantes florais no Jardim Botânico da Universidade Federal de Santa Maria, Santa Maria, RS, Brasil
}

\author{
Renata Lemes ${ }^{1 *}$ \\ Camila Duarte Ritter ${ }^{1}$ \\ Ana Beatriz Barros de Morais ${ }^{2}$ \\ ${ }^{1}$ Curso de Ciências Biológicas, Centro de Ciências Naturais e Exatas \\ Universidade Federal de Santa Maria (UFSM) \\ ${ }^{2}$ Departamento de Biologia, CCNE, UFSM \\ Faixa de Camobi, Km 09, CEP 97105-900, Santa Maria, RS, Brasil \\ * Autora para correspondência \\ renatalemes13@gmail.com
}

Submetido em 04/04/2008

Aceito para publicação em 31/08/2008

\section{Resumo}

Ambientes urbanos como parques e jardins podem oferecer muitos recursos alimentares, além de abrigo e condições favoráveis para a sobrevivência de borboletas. O presente trabalho teve como objetivo fazer um inventário das borboletas visitantes florais e das plantas visitadas por esses insetos no Jardim Botânico da Universidade Federal de Santa Maria (UFSM). No período de março de 2006 a março de 2007, foram feitas $2 \mathrm{~h}$ de observações semanais das borboletas visitantes florais. Em 108h de observação, foram registradas 1114 visitas de 39 espécies de borboletas, associadas a 43 espécies de plantas (21 famílias). Nymphalidae teve a maior riqueza de espécies $(S=18)$, seguida de Hesperiidae $(S=8)$, Pieridae $(S=7)$, Papilionidae $(S=4)$ e Lycaenidae ( $\mathrm{S}=2$ ). O pierídeo Phoebis philea philea foi a espécie mais freqüente (187 visitas), seguida dos hesperídeos Urbanus proteus proteus (100), U. teleus (73) e do ninfalídeo Heliconius erato phyllis (71). Lantana camara (Verbenaceae), Eupatorium laevigatum (Asteraceae), Russelia equisetiformis (Scrophulariaceae) e Stachytarpheta cayennensis (Verbenaceae) foram as plantas mais visitadas. O Jardim Botânico da UFSM é um exemplo de parque urbano que parece possuir recursos florais para alimentação de várias espécies de borboletas, sendo também potencial refúgio para espécies de áreas florestais do entorno.

Unitermos: borboleta, interações inseto-planta, parques urbanos.

\section{Abstract}

Butterflies (Lepidoptera: Papilionoidea and Hesperioidea) visiting flowers in the Botanical Garden of the Federal University of Santa Maria, Santa Maria, RS, Brazil. Urban environments, such as parks and gardens, may offer many alimentary resources, besides shelter and favorable conditions, for butterfly survival. This study aimed to make an inventory of butterflies visiting flowers in the Botanical Garden of the Federal University of Santa Maria (UFSM). From March 2006 to March 2007, the floral visitors were observed weekly for $2 \mathrm{~h}$. After 108 hours' observations, 1114 visits by 39 butterfly species, associated with 43 plant species (21 families), were confirmed. Among the butterflies, Nymphalidae had the highest richness of species $(S=18)$, followed by Hesperiidae $(S=8)$, Pieridae $(S=7)$, Papilionidae $(S=4)$ and Lycaenidae 
$(\mathrm{S}=2)$. The pierid Phoebis philea philea was the most frequent species (187 visits), followed by hesperiids Urbanus proteus proteus (100), U. teleus (73) and the nymphalid Heliconius erato phyllis (71). Lantana camara (Verbenaceae), Eupatorium laevigatum (Asteraceae), Russelia equisetiformis (Scrophulariaceae) and Stachytarpheta cayennensis (Verbenaceae) were the most visited plants. The Botanical Garden of UFSM is an example of an urban park that seems to provide floral resources for the feeding of many butterfly species, being also a potential refuge for species from forest areas nearby.

Key words: butterfly, insect-plant interactions, urban parks

\section{Introdução}

A Ordem Lepidoptera possui 146.277 espécies descritas e 255.000 estimadas no mundo (Heppner, 1991), sendo constituída por borboletas (13\%) e mariposas (87\%). As borboletas, cujos adultos são diurnos e coloridos, são bastante representativas no neotrópico, que possui em torno de 7.100 (Beccaloni e Gaston, 1995) a 7.900 espécies (Heppner, 1991; Lamas, 2004) nessa região. O Brasil possui uma grande diversidade desses insetos, representando quase metade das espécies neotropicais, com 3.100 (Beccaloni e Gaston, 1995) a 3.200 espécies (Brown Jr. e Freitas, 1999).

Ambientes urbanos como parques e jardins podem oferecer muitos recursos alimentares (tecidos vegetais e néctar), além de abrigo e condições favoráveis para a sobrevivência de borboletas, como níveis adequados de umidade e luminosidade (Brown Jr. e Freitas, 2002; Koh e Sodhi, 2004). Sendo assim, esses insetos podem se tornar fiéis a tais hábitats, desempenhando um importante papel na polinização de algumas espécies de plantas.

Os Jardins Botânicos são considerados, primariamente, locais de preservação de espécies vegetais "ex-situ" (Primack e Rodrigues, 2001), o que implica também na manutenção e preservação de diversas espécies animais associadas às mesmas. Além disso, tais jardins possuem um importante papel de conscientização e educação ambiental para o público visitante (Primack e Rodrigues, 2001).

O presente trabalho teve como objetivo fazer um inventário das borboletas visitantes florais e das plantas visitadas por esses insetos no Jardim Botânico da Universidade Federal de Santa Maria (UFSM).

\section{Material e Métodos}

O Jardim Botânico da Universidade Federal de Santa Maria, UFSM, localiza-se no município de Santa
Maria, RS, (29 $42^{\prime}$ S e $\left.53^{\circ} 42^{\prime} \mathrm{W}\right)$, dentro do campus Camobi, a uma altitude aproximada de $95 \mathrm{~m}$. O clima da região é subtropical úmido, com temperatura média anual de $19,2^{\circ} \mathrm{C}$ e precipitação pluvial média anual de $1708 \mathrm{~mm}$ (Maluf, 2000). O Jardim Botânico possui uma área de 13 ha, onde estão presentes 515 espécies de plantas de 96 famílias, principalmente angiospermas. Dentro dessa área encontram-se trechos de vegetação herbácea, capoeiras, canteiros de plantas medicinais, árvores frutíferas, espécies ornamentais, bambuzais e um pequeno córrego. Alguns trechos, principalmente próximos à entrada, são manejados periodicamente por via mecânica (trator) enquanto outros são manejados manualmente, e outros sofrem interferência mínima.

No período de março de 2006 a março de 2007, foram feitas duas amostragens semanais, com duração de 1 hora cada, nos horários de maior atividade das borboletas, entre as $9 \mathrm{~h}$ e $15 \mathrm{~h}$. As observações foram feitas alternadamente dentro dessa faixa de horário para não privilegiar algumas espécies em detrimento de outras. Em cada amostragem era percorrida a mesma trilha, com aproximadamente $300 \mathrm{~m}$ de extensão, localizada próxima à entrada do Jardim Botânico, onde todas as borboletas visitando flores eram contadas, por planta, e identificadas. A identificação foi feita no próprio local através de literatura especializada (Brown Jr., 1992; Canals, 2000 e 2003), a olho nu, ou com auxílio de rede entomológica, com posterior liberação dos indivíduos coletados. Todas as plantas visitadas foram marcadas e observadas uma única vez em cada ocasião amostral, durante o percurso do trajeto.

A nomenclatura das espécies de borboletas foi atualizada de acordo com Lamas (2004) e a das plantas de acordo com o APG II (Souza e Lorenzi, 2005). A maioria das espécies de plantas possuía placas de identificação no próprio local e aquelas não identificadas no campo tiveram um ramo coletado e herborizado, para consulta posterior ao Herbário da UFSM e/ou especialistas. Cada planta 
visitada pelas borboletas teve sua fenologia acompanhada até o final do estudo, de acordo com os seguintes critérios: com flores e com frutos verdes e/ou maduros, para verificar uma possível associação entre produção de frutos e freqüência de visitação por borboletas.

\section{Resultados e Discussão}

Em 108 horas de observação, foram registradas 1.114 visitas florais no Jardim Botânico da UFSM e 39 espécies de borboletas foram identificadas em 892 visitas (Tabela 1). Outros visitantes florais, como abelhas e vespas (Hymenoptera), moscas (Diptera) e beija-flores (Trochiliformes) também foram observados.

TABELA 1: Borboletas visitantes florais no Jardim Botânico da Universidade Federal de Santa Maria, Santa Maria, RS, 2006-2007, ( $\mathrm{S}=$ riqueza; $\mathrm{N}$ = número de visitas por planta.).

\begin{tabular}{|c|c|c|c|}
\hline Família & Subfamília & Espécies & $\mathbf{N}$ \\
\hline \multirow{18}{*}{ Nymphalidae } & \multirow{7}{*}{ Heliconiinae } & Agraulis vanillae maculosa (Stichel, [1908]) & 29 \\
\hline & & Dione juno juno (Cramer, 1779) & 5 \\
\hline & & Dione moneta Hübner, [1825] & 2 \\
\hline & & Dryadula phaetusa (Linnaeus, 1758) & 38 \\
\hline & & Dryas iulia alcionea (Cramer, 1779) & 50 \\
\hline & & Heliconius erato phyllis (Fabricius, 1775) & 70 \\
\hline & & Heliconius ethilla narcaea Godart, 1819 & 17 \\
\hline & \multirow{6}{*}{ Nymphalinae } & Anartia amathea roeselia (Eschscholtz, 1821) & 35 \\
\hline & & Eresia lansdorfi (Godart, 1819) & 2 \\
\hline & & Junonia evarete (Cramer, 1779) & 20 \\
\hline & & Ortilia ithra (W.F. Kirby, 1900) & 3 \\
\hline & & Siproeta epaphus trayja Hübner, [1823] & 1 \\
\hline & & Vanessa braziliensis (Moore, 1883) & 21 \\
\hline & \multirow{2}{*}{ Limenitidinae } & Adelpha sp. Hübner, [1819] & 1 \\
\hline & & Adelpha zea (Hewitson, 1850) & 2 \\
\hline & Ithomiinae & Epityches eupompe (Geyer, 1832) & 2 \\
\hline & Danainae & Danaus erippus (Cramer, 1775) & 1 \\
\hline & Satyrinae & Hermeuptychia hermes (Fabricius, 1775) & 5 \\
\hline \multirow{8}{*}{ Hesperiidae } & \multirow{8}{*}{ Pyrginae } & Achlyodes mithridates thraso (Hübner, [1807]) & 4 \\
\hline & & Codatractus aminias (Hewitson, 1867) & 18 \\
\hline & & Heliopetes omrina (Butler, 1870) & 2 \\
\hline & & Pyrgus orcus (Stoll, 1780) & 21 \\
\hline & & Urbanus dorantes dorantes (Stoll, 1790) & 50 \\
\hline & & Urbanus proteus proteus (Linnaeus, 1758) & 100 \\
\hline & & Urbanus simplicius (Stoll, 1790) & 49 \\
\hline & & Urbanus teleus (Hübner,[1821]) & 73 \\
\hline \multirow{7}{*}{ Pieridae } & \multirow{5}{*}{ Coliadinae } & Eurema sp. Hübner, [1819] & 1 \\
\hline & & Eurema elathea elathea (Cramer, 1777) & 2 \\
\hline & & Phoebis neocypris neocypris (Hübner, [1823]) & 10 \\
\hline & & Phoebis philea philea (Linnaeus, 1763) & 187 \\
\hline & & Phoebis sennae marcellina (Cramer, 1777) & 46 \\
\hline & Dismorphiinae & Enantia lina (Herbst, 1792) & 3 \\
\hline & Pierinae & Ascia monuste (Linnaeus, 1764) & 8 \\
\hline \multirow{4}{*}{ Papilionidae } & \multirow{4}{*}{ Papilioninae } & Battus polydamas polydamas (Linnaeus, 1758) & 3 \\
\hline & & Battus polystictus polystictus (Butler, 1874) & 1 \\
\hline & & Heraclides astyalus astyalus (Godart, 1819) & 1 \\
\hline & & Heraclides thoas brasiliensis (Rothschild \& Jordan, 1906) & 7 \\
\hline \multirow{2}{*}{ Lycaenidae } & \multirow{2}{*}{ Theclinae } & Calycopis caulonia (Hewitson, 1877) & 1 \\
\hline & & Parrhasius sp. Hübner, [1819] & 1 \\
\hline Total & & & 892 \\
\hline
\end{tabular}


A família de borboletas mais freqüente foi Nymphalidae (458; 41\%), seguida de Hesperiidae (374; $33,6 \%$ do total), Pieridae (257; 23,1\%), Lycaenidae (13; $1,2 \%$ ) e Papilionidae (12;1,1\%) (Figura 1A). A baixa freqüência de Lycaenidae pode estar relacionada ao fato desses insetos se caracterizarem por populações pequenas e de difícil captura (Brown Jr. e Freitas, 1999), ou por possuírem preferência alimentar diferenciada já que armadilhas com iscas de carne em decomposição são a forma mais eficaz de atraí-los (Freitas et al., 2003).

Das 222 borboletas não identificadas até o nível de espécie, 154 pertencem ao gênero Actinote (Família Nymphalidae), 57 a Hesperiidae e 11 a Lycaenidae. As espécies de Actinote são muito semelhantes e pertencem ao mesmo anel mimético (Francini e Penz, 2006) e apenas quatro espécies foram registradas anteriormente no campus da UFSM (Sackis e Morais, 2008). Assim como a maioria dos Hesperiidae e Lycaenidae, essas borboletas necessitariam ser coletadas, sacrificadas e enviadas a especialistas para sua identificação; por esse motivo optamos por privilegiar apenas a observação do comportamento desses indivíduos.

Nymphalidae foi a família de borboletas com a maior riqueza de espécies identificadas (46,1\% do total), seguida de Hesperiidae (20,5\%), Pieridae (18,0\%), Papilionidae $(10,3 \%)$ e Lycaenidae $(5,1 \%)$ (Figura 1B). Esta mesma representatividade de famílias foi encontrada por Sackis e Morais (2008) no Campus Camobi da UFSM, com inversão apenas nas riquezas das famílias Papilionidae e Lycaenidae.

No presente trabalho, foram registradas $30(33,7 \%)$ das 89 espécies observadas anteriormente no campus por Sackis e Morais (2008). E quanto à composição, registrou-se a presença de seis espécies não registradas anteriormente: o ninfalídeo $D$. moneta; o hesperídeo C. aminias; o pierídeo E. lina e os papilionídeos $B$. polydamas polydamas, B. polystictus polystictus e $H$. astyalus astyalus. Cabe mencionar que esses três papilionídeos são encontrados em fragmentos de Floresta Estacional Decidual, localizados próximos ao Campus UFSM (Dessuy e Morais, 2007).

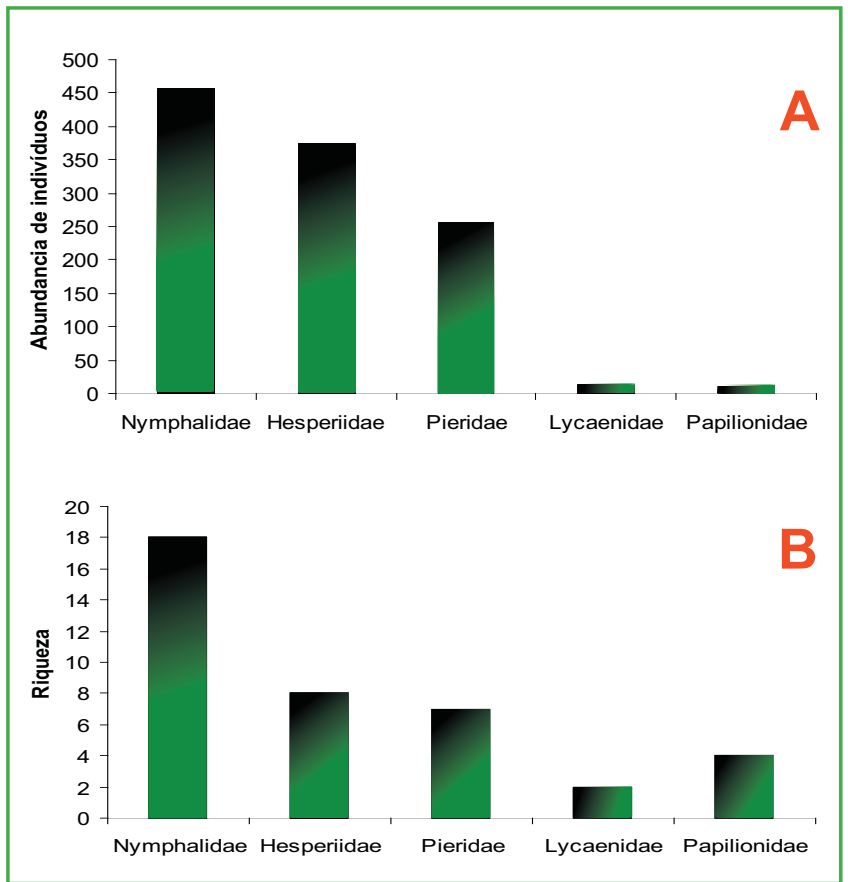

FIGURA 1: Borboletas visitantes florais presentes no Jardim Botânico da UFSM, entre março de 2006 e março de 2007. A) número de indivíduos por família; B) número de espécies identificadas por família.

O pierídeo $P$. philea philea foi a espécie mais freqüente (187 visitas, $16,8 \%$ do total de 1.114), seguida dos hesperídeos $U$. proteus proteus $(100-9,0 \%), U$. teleus $(73-6,6 \%)$ e do ninfalídeo H. erato phyllis $(71$ $-6,3 \%$ ) (Tabela 1). Representantes dessas três famílias estão entre os grupos mais comuns de borboletas visitantes florais nas regiões tropicais (De Vries, 1987; Correa et al., 2001; Fonseca et al., 2006; Kunte, 2008) e algumas espécies são consideradas polinizadoras, como U. teleus em flores de Stachytarpheta em mata atlântica (Barbola et al., 2006). No presente trabalho, essa mesma espécie de borboleta foi observada visitando plantas desse mesmo gênero.

Phoebis philea philea é observada regularmente visitando flores de várias espécies de plantas do campus da UFSM (dados não publicados). Espécies desse gênero possuem hábito migratório e vôo rápido (De Vries, 1987) o que poderia conferir-lhes uma maior necessidade energética em relação às outras borboletas. Associado a isso, o porte médio e probóscide alongada dos pierídeos lhes permite explorar uma gama maior de flores e, assim, encontrar fontes abundantes de néctar (Corbet, 2000). 
Urbanus proteus proteus é comum em campos baldios e suas lagartas se alimentam de plantas da família Fabaceae, sendo consideradas pragas de muitas espécies cultivadas (Brown Jr., 1992). Neste estudo, esse hesperídeo foi registrado visitando 17 espécies de plantas, sendo uma espécie muito comum no campus da UFSM (Sackis e Morais, 2008), em cuja área bastante antropizada encontram-se muitas espécies de plantas invasoras e lavouras experimentais (Ethur et al., 1995; Dambros et al., 2004).

Heliconius erato phyllis é considerada uma das espécies mais abundantes no Rio Grande do Sul (Morais et al., 2007), apesar de encontrar-se no seu limite sul de distribuição (Brown Jr., 1981), sendo considerada uma espécie oportunista em relação aos recursos florais utilizados e com papel potencial em relação à polinização de algumas das espécies visitadas (Correa et al., 2001). Essa borboleta é bastante comum no campus (Sackis e Morais, 2008) e no presente trabalho visitou 17 espécies das plantas observadas.

Dentre as 43 espécies de plantas identificadas, Lantana camara L. (Verbenaceae), Eupatorium laevigatum Lam. (Asteraceae), Russelia equisetiformis Schl. et Cham. (Scrophulariaceae) e Stachytarpheta cayennensis (Rich.)Vahl (Verbenaceae) foram as plantas mais visitadas, com totais de 224 (20\%), 127 (11\%), 81 $(7 \%)$ e $60(5 \%)$, respectivamente (Tabela 2). A maioria dessas espécies vegetais é nativa, de hábito arbustivo, e algumas possuem flores que se encaixam na síndrome de psicofilia (cores vivas, principalmente vermelho e laranja, odor leve, porte ereto, nectários grandes contidos em estruturas tubiformes ou esporões florais, simetria radial e borda da corola grande) (Faegri e Van Der Pij1, 1980). Essas espécies já foram observadas sendo visitadas por borboletas em áreas naturais e parques urbanos (Correa et al., 2001; Brown Jr. e Freitas, 2002; Fonseca et al., 2006).

Na maioria dos casos, não parece haver associação ou preferência entre famílias de borboletas e plantas visitadas, com exceção de $R$. equisetiformis. Esta espécie, exótica e ornamental, é conhecida como "flor-de-coral" ou "lágrima-do-diabo", e difere do padrão anteriormente descrito para psicofilia, pois suas flores tubulares vermelhas estão mais de acordo com a descrição da síndrome de ornitofilia (Faegri e Van Der Pijl, 1980). Durante a realização desse trabalho, essa espécie foi visitada exclusivamente por borboletas Pieridae do gênero Phoebis e beija-flores.

Sempre houve disponibilidade de flores no Jardim Botânico da UFSM durante os meses de observação, com um número mínimo de nove a um máximo de 21 espécies de plantas floridas por mês (Figura 2). Já o número de visitas florais variou numa amplitude mensal muito maior (3 a 337) (Figura 2). No entanto, de modo geral, não pareceu haver uma relação direta entre o número de plantas floridas e o número de visitas de borboletas. Um exemplo disso ocorreu no mês de abril de 2006, quando houve o maior número de visitas (337) em 18 plantas floridas, apenas duas a mais do que no mês de setembro, mês do menor número de visitas de borboletas (3). Nesse mês, outros fatores, que não a disponibilidade de flores, podem ter afetado a presença das borboletas no Jardim Botânico, pois sabe-se que devido a seus hábitos oportunistas e generalistas em relação aos recursos florais (Correa et al., 2001), elas conseguem explorar todos os recursos disponíveis.

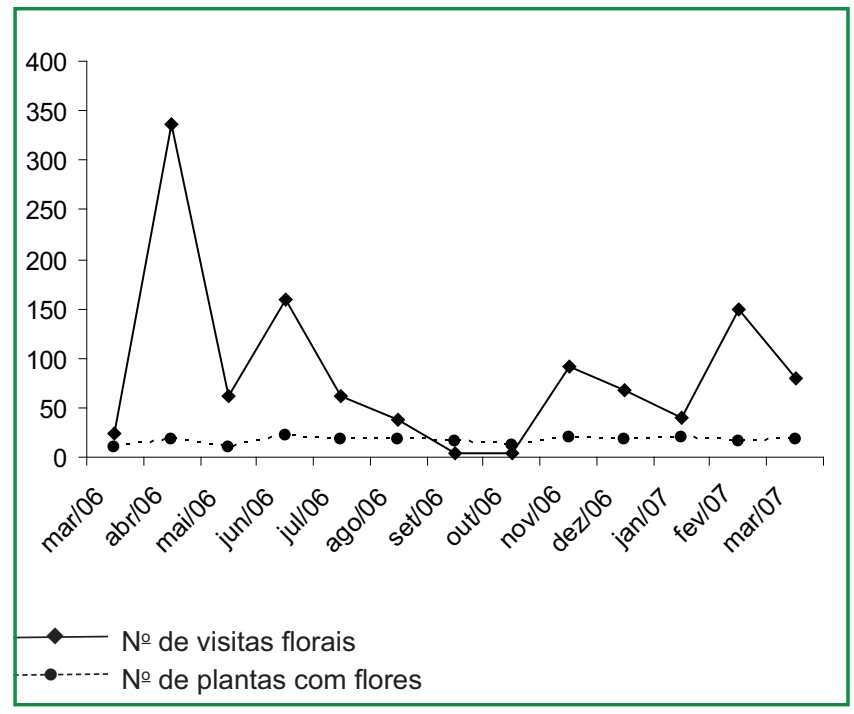

FIGURA 2: Número de borboletas visitantes florais e número de plantas com flores por mês no Jardim Botânico da UFSM, no período de março de 2006 a março de 2007. 
TABELA 2: Número de visitantes florais por espécie de plantas no Jardim Botânico da Universidade Federal de Santa Maria, Santa Maria, RS, 2006-2007.

\begin{tabular}{|c|c|c|c|}
\hline Família/Espécie & $\mathbf{N}$ & Família/Espécie & $\mathbf{N}$ \\
\hline Apocynaceae & & Melastomataceae & \\
\hline Nerium oleander L. & 5 & Tibouchina grandifolia Cogn. & 1 \\
\hline Thevitia peruviana $($ Pers)K.Schum & 31 & Tibouchina granulosa (Desr.) Cogn. & 3 \\
\hline Asteraceae & & Myrtaceae & \\
\hline Baccharis articulata (Lam.) Pers. & 2 & Melaleuca leucodendrom (L.) L. & 58 \\
\hline Cynara scolymus L. & 1 & Oleaceae & \\
\hline Elephantopus mollis H.B.K & 6 & Jasminum mesnyi Hance & 40 \\
\hline Eupatorium laevigatum Lam. & 127 & Oxalidaceae & \\
\hline Mikania micrantha H.B.K & 24 & Oxales sp. & 3 \\
\hline Bignoniaceae & & Proteaceae & \\
\hline Pyrostegia venusta Miers. & 1 & Grevillea robusta A. Cum. & 1 \\
\hline Boraginaceae & & Rosaceae & \\
\hline Cordia verbenaceae DC. & 3 & Chaenomeles speciosa Nakai & 21 \\
\hline Convolvulaceae & & Eriobotrya japonica $\mathrm{L}$ dl. & 17 \\
\hline Ipomoea sp. & 35 & Prunus persica Sieb.et Zucc. & 4 \\
\hline Ericaceae & & Pyracantha coccinea M. Roem & 30 \\
\hline Rhododendrom simsii Planch. & 23 & Rubus rosaefolius $\mathrm{Sm}$. & 47 \\
\hline Fabaceae & & Rubiaceae & \\
\hline Bauhinia variegata $\mathrm{L}$. & 20 & Gardenia jasminoides Ellis. & 3 \\
\hline Calliandra brevipes Benth. & 19 & Ixora coccinea Lam. & 21 \\
\hline Calliandra tweedii Benth. & 2 & Scrophulariaceae & \\
\hline Mimosa pudica $\mathrm{L}$. & 1 & Russelia equisetiformis Schl. et Cham. & 81 \\
\hline Iridaceae & & Solanaceae & \\
\hline Alophia sp. & 2 & Brunfelsia uniflora (Pohl) D.Don. & 8 \\
\hline Labiatae & & Solanum paniculatum L. & 4 \\
\hline Marsypianthus chamaedrys (Vahl) Kuntz. & 45 & Sterculiaceae & \\
\hline Mentha piperita $\mathrm{L}$. & 5 & Waltheria douradinha St.Hil. & 44 \\
\hline Salvia microphylla Kunth & 4 & Theaceae & \\
\hline Lamiaceae & & Camellia sinensis (L.) O. Kuntze & 36 \\
\hline Physostegia virginiana Benth. & 13 & Verbenaceae & \\
\hline Liliaceae & & Lantana camara $\mathrm{L}$. & 224 \\
\hline Aloe arborescens Mill. & 5 & Stachytarpheta cayennensis (Rich.) Vahl. & 60 \\
\hline Malvaceae & & Zingiberaceae & \\
\hline Hibiscus rosa-sinensis L. & 31 & Hedychium coronaio Koening & 3 \\
\hline \multicolumn{3}{|l|}{$\mathrm{N}$ total } & 1114 \\
\hline
\end{tabular}


Em relação à fenologia dos vegetais, $14 \%$ se mantiveram floridos por, no mínimo, 10 meses e $58 \%$ das espécies apresentaram formação de frutos. Dentre as plantas mais visitadas, todas permaneceram floridas durante todo o período de observação, exceto E. laevigatum que esteve florida somente no mês de junho, quando foi visitada pelas borboletas Actinote (que corresponderam a 59\% das visitas nessa espécie de planta). Depois de permanecer com frutos até o mês de agosto de 2006, a planta foi cortada e eliminada das observações. Quanto as outras espécies, formaramse frutos em L. camara e $S$. caynnensis e apenas $R$. equisetiformis não formou frutos durante o período de observações.

A cidade de Santa Maria está localizada na área de abrangência do Bioma Pampa e na região limite de distribuição da floresta atlântica, no entanto ainda é carente de áreas legais de preservação natural. O Jardim Botânico da UFSM é um exemplo de parque urbano que parece possuir recursos florais para alimentação de várias espécies de borboletas, sendo também potencial refúgio para espécies de áreas florestais do entorno. O presente estudo corrobora o papel de áreas urbanas na conservação de espécies animais e suas interações com os vegetais ali presentes, podendo servir como fonte de subsídios para estudos de manejo e incremento da diversidade local. O plantio de plantas hospedeiras de imaturos de borboletas e o aumento de outros recursos do ambiente como umidade, por exemplo, poderia atrair e sustentar um maior número de espécies.

\section{Agradecimentos}

À Dra Thaís S. do Canto-Dorow e à Dr ${ }^{\mathrm{a}}$ Jumaida M. Rosito (UFSM) pela gentileza da identificação das plantas. Ao coordenador do Jardim Botânico da UFSM Renato Záchia pela permissão ao acesso do mesmo e ao técnico administrativo Alberi da Silva pelo apoio durante os trabalhos de campo. A Cristian S. Dambros pela ajuda nos mais diversos momentos do trabalho. Aos dois revisores pelas pertinentes sugestões e correções deste trabalho.

\section{Referências}

Barbola, I. F.; Laroca, S.; Almeida, M. C.; Nascimento, E. A. 2006. Floral biology of Stachytarpheta maximiliani Scham. (Verbenaceae) and its floral visitors. Revista Brasileira de Entomologia, 50(4): 498-504.

Beccaloni, G. W.; Gaston, K. J. 1995. Predicting species richness of neotropical forest butterflies: Ithomiinae (Lepidoptera: Nymphalidae) as indicators. Biological Conservation, 71: 77-86.

Brown Jr., K. S. 1981. The biology of Heliconius and related genera. Annual Review of Entomology, 26: 427-456.

Brown Jr., K. S. 1992. Borboletas da Serra do Japi: diversidade, habitats, recursos alimentares e variação temporal. In: Morellato, L. P. C. (org). História natural da Serra do Japi: Ecologia e preservação de uma área florestal no sudeste do Brasil. Editora da UNICAMP, Campinas, Brasil, p.142-186.

Brown Jr., K. S.; Freitas, A. V. L. 1999. Lepidoptera. In: Brandão, C. R. F. \& Cancello, E. M. (eds). Biodiversidade do Estado de São Paulo, Brasil. Invertebrados terrestres. FAPESP, São Paulo, Brasil, p.227-243.

Brown Jr., K. S.; Freitas, A. V. L. 2002. Butterfly communities of urban forest fragments in Campinas, São Paulo, Brazil: structure, instability, environmental correlates, and conservation. Journal of Insect Conservation, 6: 217-231.

Canals, G. R. 2000. Mariposas bonaerenses. L.O.L.A., Buenos Aires, Argentina, 347pp.

Canals, G. R. 2003. Mariposas de Misiones. L.O.L.A., Buenos Aires, Argentina, 492pp.

Corbet, S. A. 2000. Butterfly nectaring flowers: butterfly morphology and flower form. Entomologia Experimentalis et Applicata, 96: $289-298$

Correa, C. A.; Irgang, B. E.; Moreira, G. R. P. 2001. Estrutura floral das angiospermas usadas por Heliconius erato phyllis (Lepidoptera, Nymphalidae) no Rio Grande do Sul, Brasil. Iheringia, Série Zoologia, 90 (1): 71-84.

Dambros, V. S.; Eisinger, S. M.; Canto-Dorow, T. S. 2004. Leguminosae do campus da Universidade Federal de Santa Maria, Rio Grande do Sul, Brasil. Ciência e Natura, 26 (2): 43-60.

Dessuy, M. B.; Morais, A. B. B. 2007. Diversidade de borboletas (Lepidoptera, Papilionoidea e Hesperioidea) em fragmentos de Floresta Estacional Decidual em Santa Maria, Rio Grande do Sul, Brasil. Revista Brasileira de Zoologia, 24 (1): 108-120.

De Vries, P. J. 1987. The butterflies of Costa Rica and their natural history: Papilionidae, Pieridae, Nymphalidae. Princeton University Press, Princeton, EUA, 327pp.

Ethur, L. Z.; Eisinger, S. M.; Ritter, M. R. 1995. Levantamento de plantas invasoras no campus da UFSM - Santa Maria, RS - Magnoliopsida. Ciência e Natura, 17: 65-73.

Faegri, K.; Van Der Pijl, L. 1980. The principles of pollination ecology. $3^{\text {rd }}$ ed. Pergamon, London, England, 244pp.

Fonseca, N. G.; Kumagai, A. F.; Mielke, O. H. H. 2006. Lepidópteros visitantes florais de Stachytarpheta cayennensis (Rich.) Vahl (Verbenaceae) em remanescente de Mata Atlântica, Minas Gerais, Brasil. Revista Brasileira de Entomologia, 50 (3): 399-405. 
Francini, R. B.; Penz, C. M. 2006. An illustrated key to male Actinote from southeastern Brazil (Lepidoptera, Nymphalidae). Biota Neotropica, 6 (1): <http://www.biotaneotropica.org.br/v6n2>.

Freitas, A. V. L.; Francini, R. B.; Brown Jr., K. S. 2003. Insetos como indicadores ambientais. In: Cullen Jr., L.; Rudran, R. \& Valladares-Padua, C. (orgs). Métodos de Estudos em Biologia da Conservação e Manejo da Vida Silvestre. Editora da Universidade Federal do Paraná, Curitiba, p.125-151.

Heppner, J. B. 1991. Faunal regions and the diversity of Lepidoptera. Tropical Lepidoptera, 2 (1): 1-85.

Koh, L. P.; Sodhi, N. S. 2004. Importance of reserves, fragments, and parks for butterfly conservation in a tropical urban landscape. Ecological Applications, 14 (6): 1695-1708.

Kunte, K. 2008. Competition and species diversity: removal of dominant species increases diversity in Costa Rican butterfly communities. Oikos, 117 (1): 69-76.

Lamas, G. (ed.). 2004. Checklist: Part 4A. Hesperioidea - Papilionoidea. In: Heppner, J. B. (ed.). Atlas of neotropical Lepidoptera.
Association for Tropical Lepidoptera/Scientific Publishers, Gainesville, USA, p. 1-439.

Maluf, J. R. T. 2000. Nova classificação climática do estado do Rio Grande do Sul. Revista Brasileira de Agrometeorologia, 8 (1): 141-150.

Morais, A. B. B.; Romanowski, H. P.; Isehard, C. A.; Marchiori, M. O. O.; Seguí, R. 2007. Mariposas del sur de Sudamérica (Lepidoptera: Hesperioidea y Papilionoidea). Revista Ciência \& Ambiente, 35: 29-46.

Primack, R. B.; Rodrigues, E. 2001. Biologia da Conservação. Midiograf, Londrina, Brasil, 327pp.

Sackis, G. D.; Morais, A. B. B. 2008. Borboletas (Lepidoptera: Hesperioidea e Papilionoidea) do campus da Universidade Federal de Santa Maria, Santa Maria, Rio Grande do Sul. Biota Neotopica, 8 (1): <http://www.biotaneotropica.org.br/v8nl >.

Souza, V. C.; Lorenzi, H. 2005. Botânica sistemática: guia ilustrado para identificação das famílias de Angiospermas da flora brasileira, baseado em APG II. Instituto Plantarum, Nova Odessa, Brasil, 639pp. 